\title{
Development of a Nomogram for Predicting the Efficacy of Preoperative Chemotherapy in Osteosarcoma
}

\author{
Qingshan Huang $\mathbb{D}^{1,2}$ \\ Chenglong Chen (D) ${ }^{1,2}$ \\ Jingbing Lou (iD) ${ }^{1,2}$ \\ Yi Huang (iD ${ }^{1,2}$ \\ Tingting Ren (D) ${ }^{1,2}$ \\ Wei Guo (iD) ${ }^{1,2}$ \\ 'Musculoskeletal Tumor Center, Peking \\ University People's Hospital, Beijing, \\ People's Republic of China; ${ }^{2}$ Beijing Key \\ Laboratory of Musculoskeletal Tumor, \\ Peking University People's Hospital, \\ Beijing, People's Republic of China
}

Background: Due to the obvious heterogeneity of osteosarcoma, many patients are not sensitive to neoadjuvant chemotherapy. In this study, the clinical characteristics and auxiliary examinations of patients with osteosarcoma were used to predict the effect of preoperative chemotherapy, so as to guide the clinical adjustment of the treatment plan to improve the prognosis of patients.

Methods: In this study, 90 patients with pathologically confirmed osteosarcoma were included, and they were randomly divided into training cohort $(n=45)$ and validation cohort $(n=45)$. A prediction model of preoperative chemotherapy efficacy for osteosarcoma was established by multivariate logistic regression analysis, and a nomogram was used as the visualization of the model. The ROC curve and C-index were used to evaluate the accuracy of the nomogram. Decision curve analysis (DCA) was used to evaluate the net benefit of the nomogram in predicting the efficacy of neoadjuvant chemotherapy under different threshold probabilities.

Results: In the study, the age, gender, location, tumor volume, metastasis at the first visit, MSTS staging, C-reactive protein (CRP), erythrocyte sedimentation rate (ESR), alkaline phosphatase (ALP), and lactate dehydrogenase (LDH) were used in the multivariate logistic regression analysis and the construction of the nomogram. The AUC and C-index of the training cohort were 0.793 (95\% CI: $0.632,0.954)$ and 0.881 (95\% CI: $0.776,0.986)$, respectively. The AUC and C-index in the validation cohort were 0.791 (95\% CI: 0.644 , 0.938 ) and 0.813 (95\% CI: $0.679,0.947)$, respectively, which were close to the training cohort. DCA showed that the model had good clinical application value.

Conclusion: Based on the clinical characteristics of patients and auxiliary examinations, the nomogram can be good used to predict the efficacy of preoperative chemotherapy for osteosarcoma.

Keywords: osteosarcoma, nomogram, chemotherapy, ROC, necrosis rate

\section{Introduction}

Osteosarcoma is the most common type of bone cancer and is commonly found in large bones in the area of bone with the fastest growth rate. ${ }^{1}$ It is a malignant bone tumor that mainly occurs in children and adolescents, ${ }^{2}$ and is the third most common tumor in adolescents. ${ }^{3}$ The advent of neoadjuvant chemotherapy has improved the prognosis of osteosarcoma, ${ }^{4}$ with the survival rate increasing from less than $20 \%$ to $60-70 \% .^{3,5,6}$ However, in recent years, the survival rate of osteosarcoma has not been further improved., 7,8 Endogenous or acquired drug resistance is one of the important causes of treatment failure and poor prognosis
Correspondence: Wei Guo

Musculoskeletal Tumor Center, Peking

University People's Hospital, Beijing,

I00044, People's Republic of China

$\mathrm{Tel} / \mathrm{Fax}+86-10-8832447$ I

Email bonettumor@163.com 
in patients with osteosarcoma. ${ }^{9}$ Osteosarcoma is one of the most heterogeneous solid tumors, ${ }^{10}$ and different patients with osteosarcoma have obvious differences in the efficacy of preoperative chemotherapy. At present, tumor cell necrosis rate of more than $90 \%$ is considered as effective chemotherapy, while tumor cell necrosis rate of less than $90 \%$ is considered as unsatisfactory chemotherapy efficacy, and endogenous drug resistance may exist. ${ }^{11,12}$

Neoadjuvant chemotherapy allows patients to achieve a tumor-free margin while preserving the limb. ${ }^{13}$ It also increases the chance of relapse-free survival in patients with osteosarcoma. ${ }^{14,15}$ Therefore, the choice of chemotherapy regimen for newly diagnosed patients is extremely important, and reasonable chemotherapy regimens are an important way to prevent the progression and metastasis of osteosarcoma. The NCCN guidelines state that standard first-line chemotherapy consists of a combination of cisplatin, doxorubicin, ifosfamide, and high-dose methotrexate. The preferred regimen is the combination of cisplatin and doxorubicin or the combination of cisplatin and doxorubicin plus high-dose methotrexate. ${ }^{4}$ Although many patients with osteosarcoma respond to standard chemotherapy regimens, there are still many patients who do not respond well to chemotherapy. We hope to predict the efficacy of chemotherapy based on the clinical characteristics of patients at the time of initial diagnosis and the relevant ancillary examination results. Therefore, chemotherapy regimens can be adjusted to improve the therapeutic effect for patients who may be ineffective. This study retrospectively analyzed the clinical, laboratory, and imaging data of 90 patients with osteosarcoma who underwent preoperative chemotherapy. The primary purpose of this study was to develop a nomogram that predicted the efficacy of preoperative chemotherapy in patients with osteosarcoma and to evaluate the probability that a patient would be effective (necrosis rate greater than $90 \%$ ).

\section{Materials and Methods}

\section{Patients}

This study was retrospective and approved by the Ethics Committee of Peking University People's Hospital. It included 90 patients with osteosarcoma who were hospitalized in the Musculoskeletal Tumor Center, Peking University People's Hospital. The patients were admitted between March 2015 and December 2018. Inclusion criteria: 1) Patients with the initial diagnosis of osteosarcoma; 2) The patient had single osteosarcoma; 3) The diagnosis was confirmed by biopsy. Exclusion criteria: 1) Patients with other malignant tumors; 2) Patients with infection; 3) Patients with incomplete clinical characteristics or auxiliary examination results; 4) Patients who did not receive neoadjuvant chemotherapy; 5) Patients undergoing radiotherapy for other reasons.

\section{Clinical Characteristics and Auxiliary Examination Items}

Clinical characteristics and auxiliary examination items, including age, gender, location, tumor volume, metastasis at the first visit, neoplasm staging, C-reactive protein (CRP), erythrocyte sedimentation rate (ESR), alkaline phosphatase (ALP), and lactate dehydrogenase (LDH) were obtained from the medical records. The tumor sites are the trunk, humerus, femur, tibia, and fibula. Musculoskeletal Tumor Society (MSTS) staging system was used for tumor staging. CRP, ESR, ALP, and LDH were divided into normal group and elevated group according to a normal reference value. The calculation method of tumor volume adopted the previous method (height $\times$ length $\times$ width $\times \pi / 6$ ). ${ }^{16-18}$ These length indicators were derived from CT imaging.

\section{Construction of the Nomogram}

The nomogram is widely used to calculate the diagnostic probability of the disease. It can reduce the complicated statistical prediction model to a probability value. ${ }^{19,20}$ A prediction model of preoperative chemotherapy efficacy for osteosarcoma was established by multivariate logistic regression analysis, and a nomogram was used as the visualization of the model. The "rms" package was used for multivariate logistic regression analysis. The regression coefficients were obtained for each variable by logistic regression analysis. They show how much each indicator contributes to the model. "rms" package was also used for the construction of the nomogram. In the study, the age, gender, location, tumor volume, metastasis at the first visit, MSTS staging, CRP, ESR, ALP, and LDH were used to construct the nomogram. It intuitively predicts the probability that preoperative chemotherapy will be effective in patients with osteosarcoma.

\section{Statistical Analysis}

$\mathrm{R}$ software (Version 3.6.1) and SPSS 22 were used for statistical analysis. Chi-square test and $t$-test were used 
to test the balance of sample distribution between the training cohort and validation cohort. The "rms" package was used for logistic regression analysis, nomogram plotting and $\mathrm{C}$-index calculation. The prediction model of preoperative chemotherapy efficacy for osteosarcoma was established by multivariate logistic regression analysis, and a nomogram was used as the visualization of the model. C-index and ROC curves were used to evaluate the accuracy of the nomogram. ${ }^{21}$ The "ROCR" package was used to plot the ROC curve and calculate the AUC value. The net benefit is defined as the proportion of true positives minus the proportion of false positives, weighted by the relative harm of false-positive and false-negative results. ${ }^{21,22}$ The "rmda" package was used to plot the decision curve and evaluate the net benefits under different threshold probabilities. Two-tailed $P$-values were used, and the statistical significance was set at $P<0.05$.

\section{Results}

\section{Patient Characteristics}

In this study, 90 patients with osteosarcoma were included, and the clinical and auxiliary examination results of each patient were shown in Figure 1. The process of patient inclusion was shown in Figure S1. The patients were arranged from small to large according to age. In this study, patients were randomly divided into the training cohort $(n=45)$ and validation cohort $(n=45)$, and all clinical characteristics and ancillary examination results of patients were summarized (Table 1). Except for $\mathrm{LDH}$, the sample size of other indicators showed no significant difference between the training cohort and the validation cohort $(\mathrm{P}>0.05)$. This indicates that the samples are evenly distributed in the two queues. There were 27 patients with good chemotherapy efficacy, accounting for 30\%. They ranged in age from 3 years to 56 years, with an average age

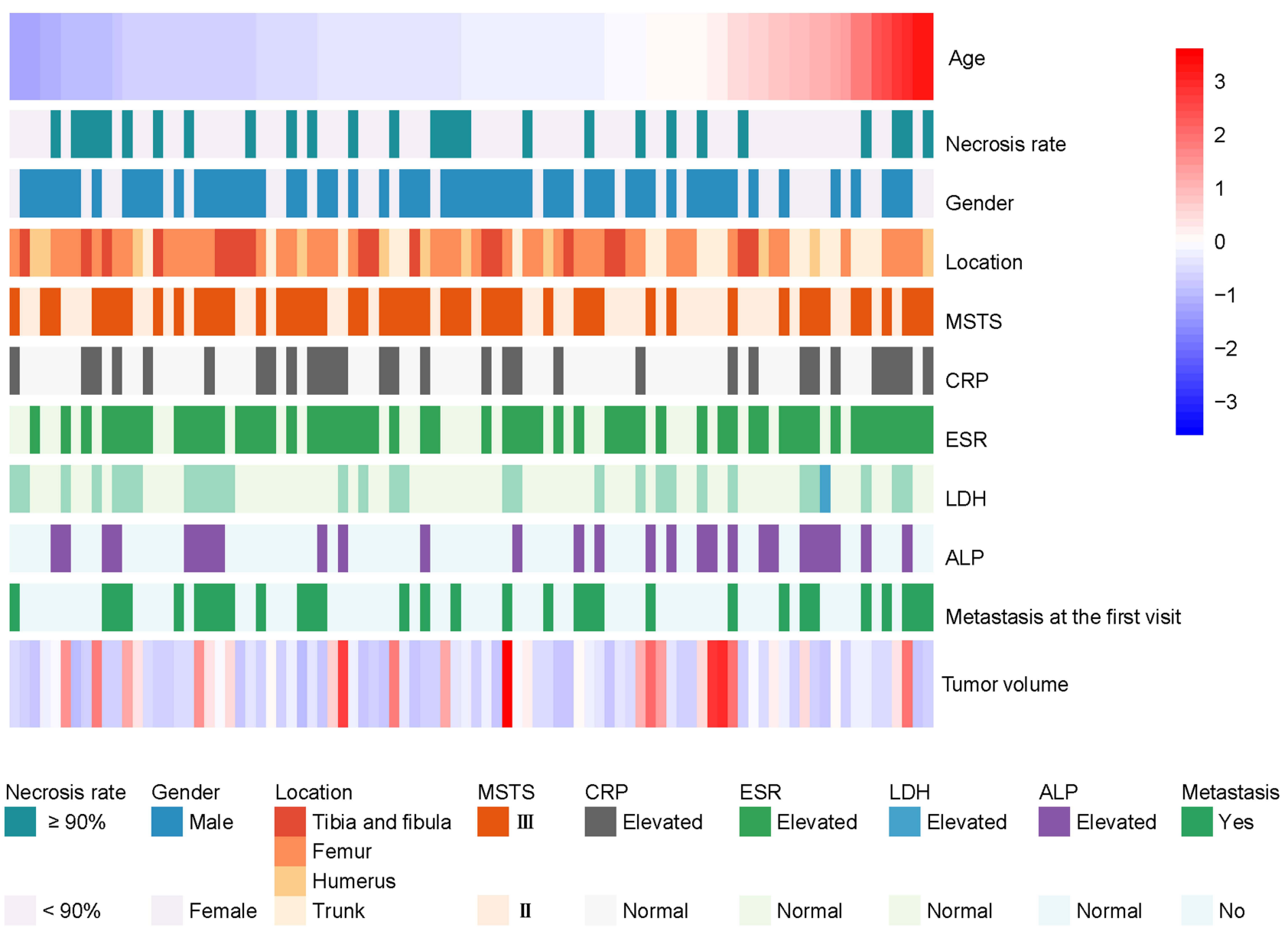

Figure I Clinical characteristics and ancillary examination results of 90 patients with osteosarcoma. Patients were randomly divided into the training cohort ( $\mathrm{n}=45$ ) and validation cohort $(n=45)$. Each line in the heat map represents a patient. Values for age and tumor volume were standardized. Abbreviations: ALP, alkaline phosphatase; CRP, C-reactive protein; ESR, erythrocyte sedimentation rate; LDH, lactate dehydrogenase. 
Table I Summary of Clinical Characteristics and Adjuvant Examination Items in Patients with Osteosarcoma

\begin{tabular}{|c|c|c|c|c|c|}
\hline Characteristics & Training Cohort $(n=45)$ & Validation cohort $(n=45)$ & Total $(n=90)$ & $\operatorname{tor} \chi^{2}$ & $P$-value \\
\hline \multicolumn{6}{|l|}{ Necrosis rate } \\
\hline$<90 \%$ & $33(73.3 \%)$ & $30(66.7 \%)$ & $63(70.0 \%)$ & 0.476 & 0.490 \\
\hline$\geq 90 \%$ & $12(26.7 \%)$ & $15(33.3 \%)$ & $27(30.0 \%)$ & & \\
\hline Age (mean, years) & 18.31 & 17.67 & 17.99 & 0.263 & 0.793 \\
\hline \multicolumn{6}{|l|}{ Gender } \\
\hline Female & 15 (33.3\%) & $16(35.6 \%)$ & $31(34.4 \%)$ & 0.000 & 1.000 \\
\hline Male & $30(66.7 \%)$ & 29 (64.4\%) & $59(65.6 \%)$ & & \\
\hline \multicolumn{6}{|l|}{ Location } \\
\hline Trunk & $7(15.6 \%)$ & $10(22.2 \%)$ & $17(18.9 \%)$ & 1.509 & 0.680 \\
\hline Humerus & $5(11.1 \%)$ & $6(13.3 \%)$ & $\mathrm{II}(12.2 \%)$ & & \\
\hline Femur & $22(48.9 \%)$ & $22(48.9 \%)$ & $44(48.9 \%)$ & & \\
\hline Tibia and fibula & II (24.4\%) & 7 (15.6\%) & $18(20.0 \%)$ & & \\
\hline Tumor volume (mean, $\mathrm{cm}^{3}$ ) & 407 & 389 & 398 & 0.198 & 0.844 \\
\hline \multicolumn{6}{|l|}{ MSTS staging } \\
\hline II & $24(53.3 \%)$ & 17 (37.8\%) & $4 \mathrm{l}(45.6 \%)$ & 2.195 & 0.138 \\
\hline III & 21 (46.7\%) & $28(62.2 \%)$ & 49 (54.4\%) & & \\
\hline \multicolumn{6}{|l|}{ CRP } \\
\hline Normal & 29 (64.4\%) & $30(66.7 \%)$ & $59(65.6 \%)$ & 0.049 & 0.824 \\
\hline Elevated & $16(35.6 \%)$ & 15 (33.3\%) & 31 (34.4\%) & & \\
\hline \multicolumn{6}{|l|}{ ESR } \\
\hline Normal & 14 (3I.I\%) & 17 (37.8\%) & 31 (34.4\%) & 0.443 & 0.506 \\
\hline Elevated & 31 (68.9\%) & $28(62.2 \%)$ & 59 (65.6\%) & & \\
\hline \multicolumn{6}{|l|}{ LDH } \\
\hline Normal & $36(80.0 \%)$ & $25(55.6 \%)$ & $61(67.8 \%)$ & 6.156 & 0.013 \\
\hline Elevated & $9(20.0 \%)$ & 20 (44.4\%) & 29 (32.2\%) & & \\
\hline \multicolumn{6}{|l|}{ ALP } \\
\hline Normal & 35 (77.8\%) & 29 (64.4\%) & $64(71.1 \%)$ & 1.947 & 0.163 \\
\hline Elevated & $10(22.2 \%)$ & $16(35.6 \%)$ & $26(28.9 \%)$ & & \\
\hline \multicolumn{6}{|l|}{ Metastasis at the first visit } \\
\hline Yes & $31(68.9 \%)$ & $28(62.2 \%)$ & 59 (65.6\%) & 0.443 & 0.506 \\
\hline No & $14(31.1 \%)$ & $17(37.8 \%)$ & 31 (34.4\%) & & \\
\hline
\end{tabular}

Note: Chi-square test and $t$-test were used to test the balance of sample distribution between training training cohort and validation cohort. Abbreviations: ALP, alkaline phosphatase; CRP, C-reactive protein; ESR, erythrocyte sedimentation rate; LDH, lactate dehydrogenase.

of $17.99 \pm 11.55$ years. The proportion of male patients was $65.6 \%$, and that of female patients was $34.4 \%$. The locations of the tumors were trunk, humerus, femur, tibia, and fibula, $48.9 \%$ of which were located in the femur. The MSTS staging showed that all the 90 patients were at stage II or stage III. Among them, most of the patients in stage II were stage II B and few were in stage II A, so they were uniformly classified as stage II in this study. At least $30 \%$ of patients have elevated CRP, ESR, LDH, and ALP. In addition, approximately $34.4 \%$ of patients had metastases at the time of their first visit.

\section{Construction of a Nomogram to Predict the Efficacy of Chemotherapy for Osteosarcoma}

In the multivariate regression analysis, the regression coefficient for intercept was -20.492 . The regression coefficients for location (humerus, femur, tibia, and fibula) were $20.866,21.839,19.935$ respectively. The 
regression coefficients for age, gender, tumor volume, metastasis, MSTS staging, CRP, ESR, LDH and ALP were $-0.064,-3.583,0.004,-0.125,2.788$, $0.872,-0.955,-2.094,-0.620$ respectively. Nomogram includes point, clinical characteristics, auxiliary examinations, total point, linear predicted value, and corresponding probability values (Figure 2). The first line showed the point range (0-100) corresponding to each clinical characteristic and auxiliary examination of the patient. Patients' clinical characteristics and ancillary examinations have corresponding points in the nomogram. The total points of all clinical characteristics and auxiliary examinations of each patient corresponds to the position on the last line, which is the probability value of effective chemotherapy. Besides, it is 9.27 points per unit of the linear predictor.

\section{Evaluation of the Accuracy of Chemotherapy Efficacy Prediction Nomogram}

In this study, the ROC curve and C-index were used to evaluate the accuracy of the prediction nomogram. ROC curve showed that the training cohort has a good predictive performance, with an AUC of 0.793 (95\% CI: 0.632, 0.954) (Figure $3 \mathrm{~A}$ ). The optimal cut-off value of the total point was 189.839. Grouping with the optimal cut-off value, we found that the sensitivity was 0.583 , the specificity was 0.939 , the positive predictive value (PPV) was 0.778 , the negative predictive value (NPV) was 0.861 , and the accuracy was 0.844 . The AUC of the validation cohort was 0.791 (95\% CI: 0.644, 0.938) (Figure 3B), which was close to that of the training cohort. There was no difference in ROC results by comparing the training cohort and verification cohort $(\mathrm{P}=0.987)$ (Figure $3 \mathrm{C})$. Besides, the

Points

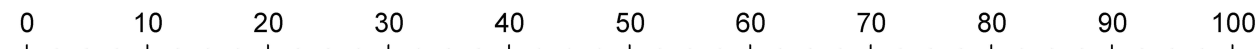

Age (years)

Gender

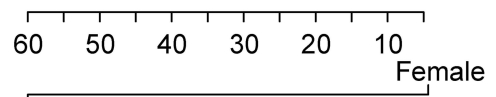

Location

Tumor volume $\left(\mathrm{cm}^{3}\right)$

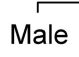
Female

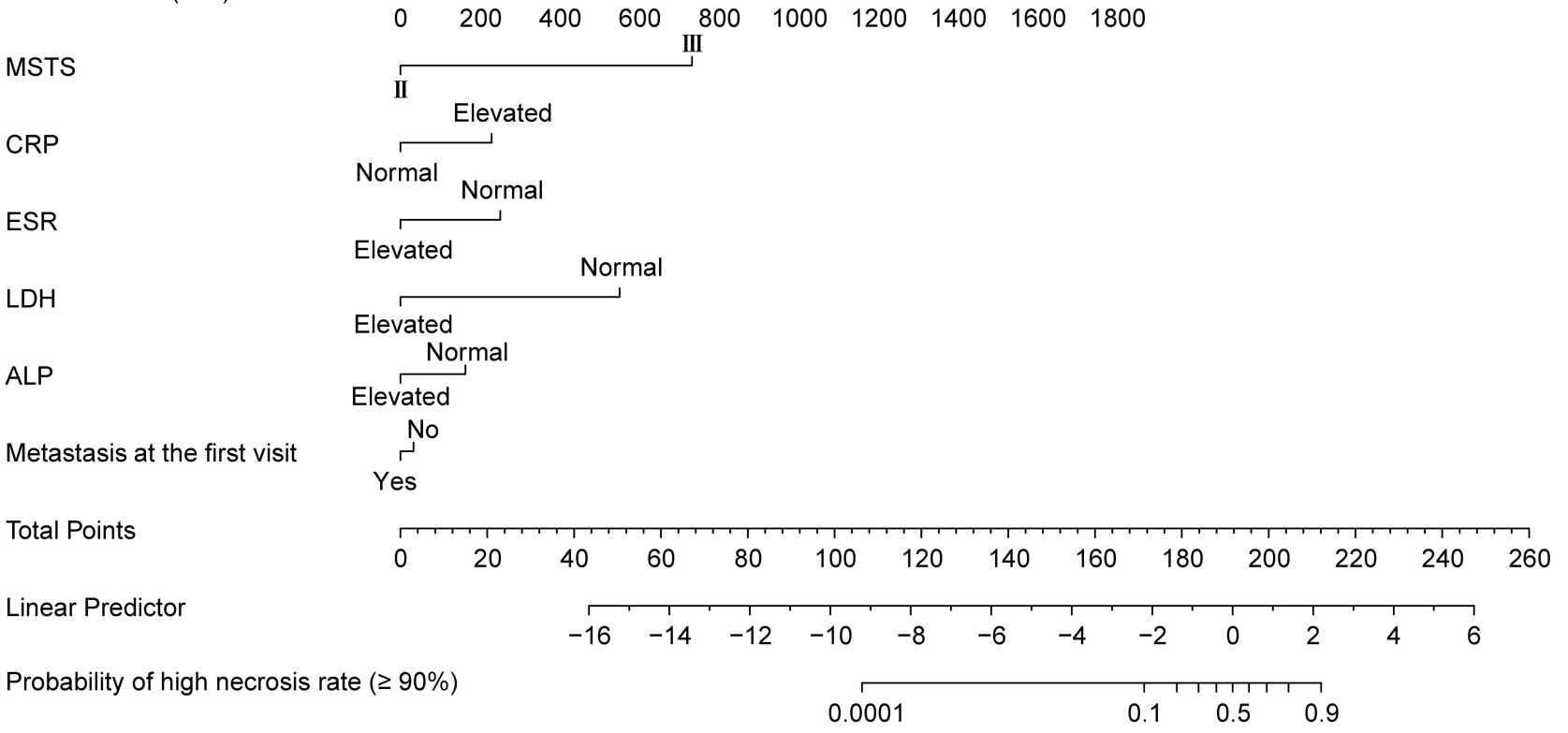

Figure 2 The nomogram to predict the efficacy of chemotherapy for osteosarcoma. The first line is the point range for the variables. The second to IIth lines are the ten clinical characteristics and ancillary examination items included in the study. The last one represents the probability value of a high necrosis rate ( $\geq 90 \%)$.

Abbreviations: ALP, alkaline phosphatase; CRP, C-reactive protein; ESR, erythrocyte sedimentation rate; LDH, lactate dehydrogenase. 
A

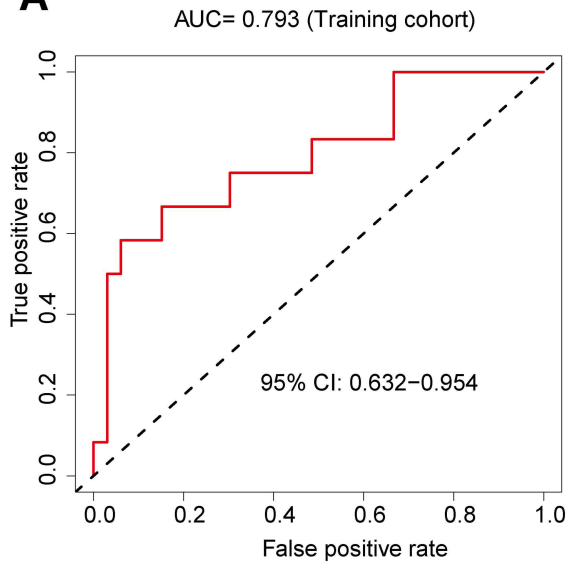

B

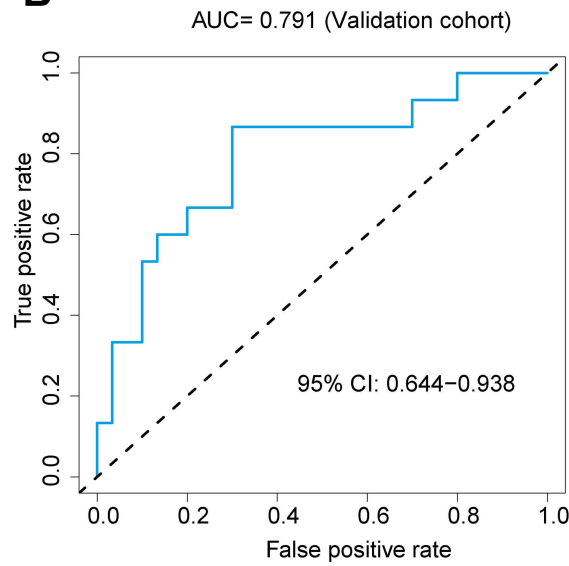

C

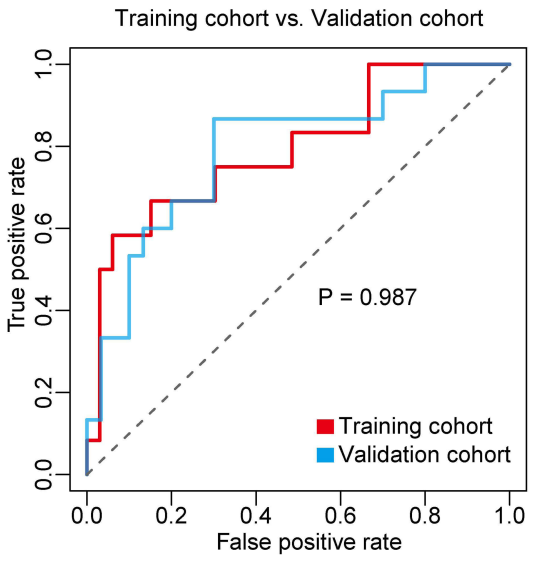

Figure 3 Evaluation of the accuracy of the chemotherapy efficacy prediction nomogram. (A) ROC is used to evaluate the predictive power of the chemotherapy efficacy prediction nomogram. The AUC in the training cohort is 0.793 ( $95 \% \mathrm{Cl}: 0.632,0.954)$. (B) The AUC in the validation cohort is $0.79 \mathrm{l}$ ( $95 \% \mathrm{Cl}$ : $0.644,0.938$ ). (C) ROC results of the training cohort and the validation cohort were compared.

C-index, Somers' $D_{x y}$ rank correlation $\left(D_{x y}\right)$, and $\mathrm{R}$-squared index $\left(\mathrm{R}^{2}\right)$ in the training cohort were 0.881 , 0.763 , and 0.536 respectively, and they were $0.813,0.627$, and 0.358 respectively in the validation cohort (Table 2).

\section{Clinical Application Value of Chemotherapy Efficacy Prediction Nomogram}

DCA was used to evaluate the potential clinical application of this nomogram. The study showed that the threshold probabilities in the training cohort were $1 \%$ and $58 \%$ (Figure 4A). That is, when the patient's individual threshold probability is between $1 \%$ and $58 \%$, there is a net benefit to using the nomogram to decide whether to receive the chemotherapy. In addition, the threshold probabilities for the validation cohort were $4 \%$ and $67 \%$ (Figure 4B).

\section{Discussion}

In this study, a nomogram was constructed to predict the efficacy of chemotherapy based on the clinical characteristics and auxiliary examination results before

Table 2 The Validation of Chemotherapy Efficacy Prediction Nomogram

\begin{tabular}{|l|l|l|}
\hline Parameter & Training Cohort & Validation Cohort \\
\hline C-index & $0.88 I$ & 0.813 \\
$D_{x y}$ & 0.763 & 0.627 \\
$R^{2}$ & 0.536 & 0.358 \\
\hline
\end{tabular}

Abbreviations: $D_{x y}$ Somers' $D_{x y}$ rank correlation; $R^{2}$, R-squared index. chemotherapy for osteosarcoma. The probability of the effectiveness of chemotherapy can be easily determined by the nomogram, so as to find out the patients who may have poor efficacy of chemotherapy in advance. Modification of treatment regimens in these patients is expected to improve patient outcomes.

Neoadjuvant chemotherapy regimens for osteosarcoma mainly include cisplatin, doxorubicin, ifosfamide, and high-dose methotrexate. ${ }^{23}$ The combination of neoadjuvant chemotherapy and surgery has greatly improved the prognosis of osteosarcoma. However, due to the presence of drug-resistant tumor cells, ${ }^{23}$ there are still $20-30 \%$ of patients who are ineffective. ${ }^{24}$ The nomogram can integrate different variables to generate the probability of each clinical event, fulfilling our need for personalized treatment. ${ }^{22}$ In the study, the age, gender, location, tumor volume, metastasis at the first visit, MSTS staging, CRP, ESR, ALP, and LDH were used to construct the nomogram. These indicators are clinically common and belong to the non-invasive examination. At present, the abnormal elevation of ALP in blood examination may have a certain suggestive effect on osteosarcoma, so it is often included in many studies. ${ }^{25,26}$ Therefore, ALP was included in our study. In addition, CRP and ESR increases are more obvious in malignant tumors, especially those with rapid growth, they are of significance for the prognosis of osteosarcoma. $^{27} \mathrm{LDH}$ is also associated with poor prognosis in patients with osteosarcoma. ${ }^{28}$ The NCCN guidelines also indicate that ALP and LDH are associated with the diagnosis and prognosis of osteosarcoma. ${ }^{4}$ These indicators are readily available at the time of hospitalization, 

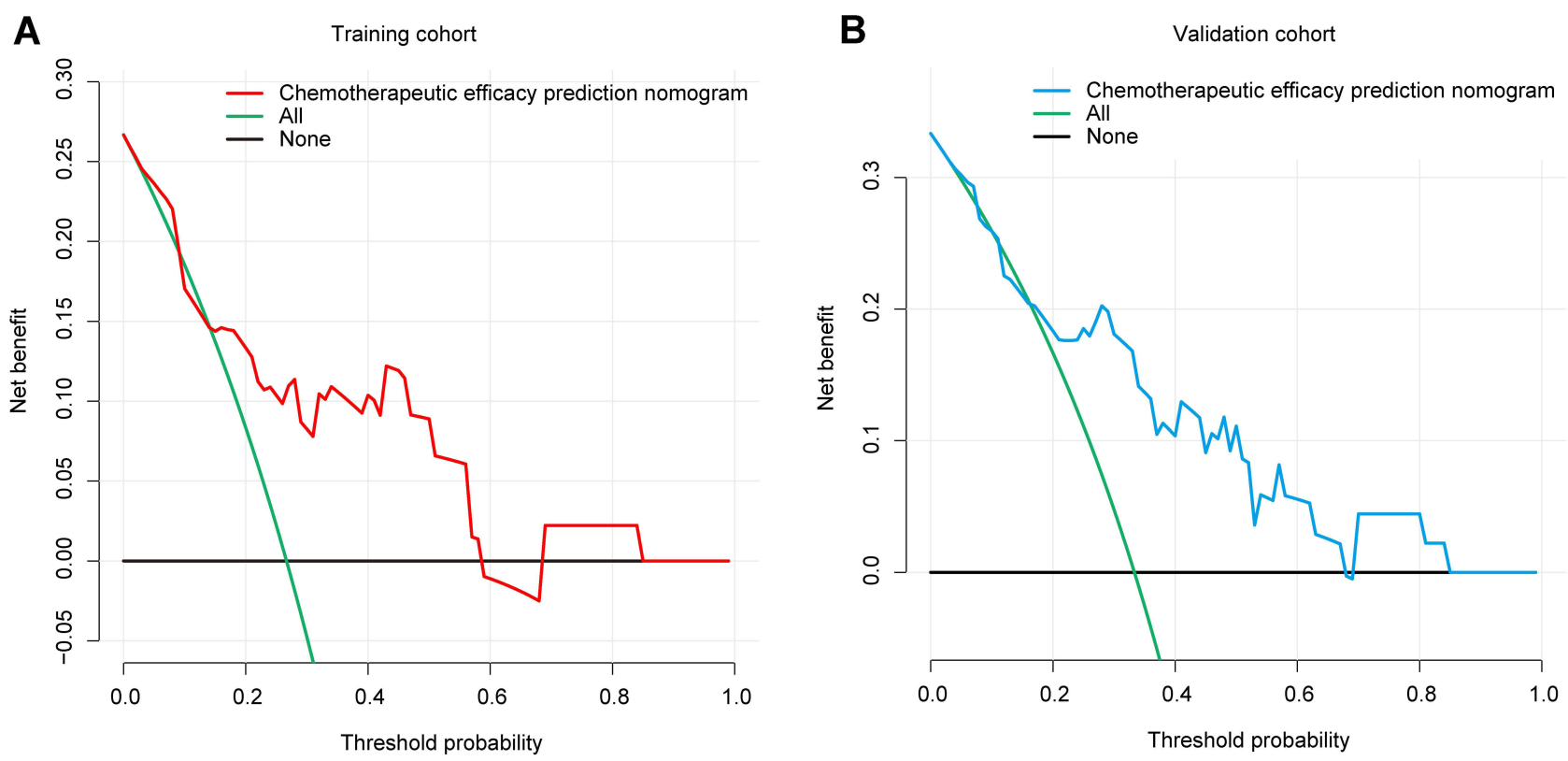

Figure 4 Decision curve analysis for the chemotherapy efficacy prediction nomogram. (A) The red line represents the chemotherapy efficacy prediction nomogram of the training cohort. When the threshold probability is between $1 \%$ and $58 \%$, the nomogram has a relatively good net benefit. (B) The blue line represents the chemotherapy efficacy prediction nomogram of the validation cohort. When the threshold probability is between $4 \%$ and $67 \%$, the nomogram has a relatively good net benefit.

so we included them in our study. The nomogram showed that the site of the tumor contributed more to the total points, and osteosarcoma in the extremities had a greater probability of responding to chemotherapy. In addition, tumor volume also contributed greatly to the total points, and the larger the tumor volume was, the higher the probability of high necrosis rate was. Of course, this may be related to insufficient blood supply caused by larger tumors. There are mainly two aspects of the quality of the general evaluation model. One is the Goodness of Fit of the model. The common evaluation index mainly includes $\mathrm{R}^{2}$. It represents the percentage change in the dependent variable that the model can explain. It is worth noting that $\mathrm{R}^{2}$ is only a reference index, not a decisive one. Another is the prediction accuracy of the model. The C-index is essentially an estimate of the probability that the prediction results will be consistent with the actual observed results. Similarly, $\mathrm{D}_{\mathrm{xy}}$ was used to evaluate the model's discrimination ability. In the study, the ROC curve and C-index were used to verify the accuracy of the nomogram, and both of them reached a high level. The validation cohort also further confirmed the reliability of the nomogram. DCA is used to evaluate the potential clinical impact of biomarkers or models and is an analytical method used to recommend treatment or intervention behavior. ${ }^{29}$ It is an appropriate method to evaluate alternative diagnostic and prognostic strategies and has advantages over other commonly used measures and techniques. ${ }^{30}$ The results of DCA in this study indicated that the nomogram for predicting the efficacy of chemotherapy for osteosarcoma had a good clinical application value.

There are some limitations to our research. This study is a retrospective study. Due to incomplete information, we could not include more indicators for statistical analysis, even though some indicators may be meaningful for the diagnosis of osteosarcoma. Second, this was a singlecenter study, and the patient origin may lead to bias in the results. In addition, the incidence of osteosarcoma is 3-4 per million, ${ }^{31}$ which is a relatively rare tumor. In the study, the strict screening was carried out according to the above exclusion criteria. Therefore, only 90 patients were included in the study, which is a weakness of this study.

In conclusion, this study presents and validates a nomogram to predict the efficacy of chemotherapy for osteosarcoma. Through the clinical characteristics and auxiliary examination results of the nomogram, we can easily predict whether the patient will have a good chemotherapy efficacy.

\section{Abbreviations}

ALP, alkaline phosphatase; CRP, C-reactive protein; $D_{x y}$, Somers' $\mathrm{D}_{\mathrm{xy}}$ rank correlation; ESR, erythrocyte sedimentation rate; $\mathrm{LDH}$, lactate dehydrogenase; $\mathrm{R}^{2}$, R-squared index. 


\section{Data Sharing Statement}

All data used in this study are included in the article.

\section{Ethics Approval and Informed Consent}

This study was approved by the Ethics Committee of Peking University People's Hospital. Due to the study was a retrospective analysis, patient consent to review their medical records was not required by the Ethics Committee of Peking University People's Hospital. All data were anonymized to comply with the provisions of personal data protection legislation. This study adhered to the tenets of the Declaration of Helsinki.

\section{Author Contributions}

All authors made a significant contribution to the work reported, whether that is in the conception, study design, execution, acquisition of data, analysis and interpretation, or in all these areas; took part in drafting, revising or critically reviewing the article; gave final approval of the version to be published; have agreed on the journal to which the article has been submitted; and agree to be accountable for all aspects of the work.

\section{Funding}

This work was supported by the Beijing Science and Technology Planning Project (No. Z161100000116100) and the National Natural Science Foundation of China (No.81572633 and No.82072970).

\section{Disclosure}

The authors declare that there is no conflict of interest regarding the publication of this paper.

\section{References}

1. National Comprehensive Cancer Network [homepage on the Internet]. NCCN guidelines for patients: bone cancer; 2021. Available from: https://www.nccn.org/. Accessed August 16, 2021.

2. Chou AJ, Geller DS, Gorlick R. Therapy for osteosarcoma: where do we go from here? Paediatr Drugs. 2008;10(5):315-327. doi:10.2165/ 00148581-200810050-00005

3. Yu X, Yustein JT, Xu J. Research models and mesenchymal/epithelial plasticity of osteosarcoma. Cell Biosci. 2021;11(1):94. doi:10.1186/ s13578-021-00600-w

4. National Comprehensive Cancer Network [homepage on the Internet]. NCCN clinical practice guidelines in oncology: bone cancer; 2021. Available from: https://www.nccn.org/. Accessed August 16, 2021.

5. Huang Q, Liang X, Ren T, et al. The role of tumor-associated macrophages in osteosarcoma progression - therapeutic implications. Cell Oncol. 2021;44(3):525-539. doi:10.1007/s13402-021-00598-w
6. Sugiu K, Tazawa H, Hasei J, et al. Oncolytic virotherapy reverses chemoresistance in osteosarcoma by suppressing MDR1 expression. Cancer Chemother Pharmacol. 2021;88(3):513-524. doi:10.1007/ s00280-021-04310-5

7. Hattinger CM, Fanelli M, Tavanti E, et al. Advances in emerging drugs for osteosarcoma. Expert Opin Emerg Drugs. 2015;20 (3):495-514. doi:10.1517/14728214.2015.1051965

8. Hattinger CM, Fanelli M, Tavanti E, et al. Doxorubicin-resistant osteosarcoma: novel therapeutic approaches in sight? Future Oncol. 2017;13(8):673-677. doi:10.2217/fon-2016-0519

9. Lin Z, Xie X, Lu S, Liu T. Noncoding RNAs in osteosarcoma: implications for drug resistance. Cancer Lett. 2021;504:91-103. doi:10.1016/j.canlet.2021.02.007

10. Schiavone K, Garnier D, Heymann MF, Heymann D. The heterogeneity of osteosarcoma: the role played by cancer stem cells. $A d v \operatorname{Exp}$ Med Biol. 2019;1139:187-200.

11. Hanafy E, Al Jabri A, Gadelkarim G, Dasaq A, Nazim F, Al Pakrah M. Tumor histopathological response to neoadjuvant chemotherapy in childhood solid malignancies: is it still impressive? J Investig Med. 2018;66(2):289-297. doi:10.1136/jim-2017-000531

12. Prudowsky ZD, Yustein JT. Recent insights into therapy resistance in osteosarcoma. Cancers. 2020;13(1):83. doi:10.3390/cancers13010083

13. Rosen G, Murphy ML, Huvos AG, Gutierrez M, Marcove RC. Chemotherapy, en bloc resection, and prosthetic bone replacement in the treatment of osteogenic sarcoma. Cancer. 1976;37(1):1-11. doi:10.1002/1097-0142(197601)37:1<1::AID-CNCR2820370102>3. $0 . \mathrm{CO} ; 2-3$

14. Link MP, Goorin AM, Miser AW, et al. The effect of adjuvant chemotherapy on relapse-free survival in patients with osteosarcoma of the extremity. $N$ Engl J Med. 1986;314(25):1600-1606. doi:10.1056/NEJM198606193142502

15. Rosen G, Caparros B, Huvos AG, et al. Preoperative chemotherapy for osteogenic sarcoma: selection of postoperative adjuvant chemotherapy based on the response of the primary tumor to preoperative chemotherapy. Cancer. 1982;49(6):1221-1230. doi:10.1002/ 1097-0142(19820315)49:6<1221::AID-CNCR2820490625>3.0. $\mathrm{CO} ; 2-\mathrm{E}$

16. Thiel DD, Jorns J, Lohse CM, Cheville JC, Thompson RH, Parker AS. Maximum tumor diameter is not an accurate predictor of renal cell carcinoma tumor volume. Scand J Urol. 2013;47 (6):472-475. doi:10.3109/21681805.2013.814071

17. MacMahon PJ, Kennedy AM, Murphy DT, Maher M, McNicholas MM. Modified prostate volume algorithm improves transrectal US volume estimation in men presenting for prostate brachytherapy. Radiology. 2009;250(1):273-280. doi:10.1148/radiol. 2501080290

18. Cander S, Oz Gul O, Eylemer E, et al. Comparison of the effects of medical and surgical treatments in giant prolactinoma: a single-center experience. Hormones. 2021;20(3):491-498. doi:10.1007/s42000021-00307-0

19. Iasonos A, Schrag D, Raj GV, Panageas KS. How to build and interpret a nomogram for cancer prognosis. J Clin Oncol. 2008;26 (8):1364-1370. doi:10.1200/JCO.2007.12.9791

20. Wang Y, Li J, Xia Y, et al. Prognostic nomogram for intrahepatic cholangiocarcinoma after partial hepatectomy. J Clin Oncol. 2013;31 (9):1188-1195. doi:10.1200/JCO.2012.41.5984

21. Huang YQ, Liang CH, He L, et al. Development and validation of a radiomics nomogram for preoperative prediction of lymph node metastasis in colorectal cancer. J Clin Oncol. 2016;34(18):21 57-2164. doi:10.1200/JCO.2015.65.9128

22. Balachandran VP, Gonen M, Smith JJ, DeMatteo RP. Nomograms in oncology: more than meets the eye. Lancet Oncol. 2015;16(4):e173e180. doi:10.1016/S1470-2045(14)71116-7

23. Isakoff MS, Bielack SS, Meltzer P, Gorlick R. Osteosarcoma: current treatment and a collaborative pathway to success. J Clin Oncol. 2015;33(27):3029-3035. doi:10.1200/JCO.2014.59.4895 
24. Takahashi N, Nobusue H, Shimizu T, et al. ROCK inhibition induces terminal adipocyte differentiation and suppresses tumorigenesis in chemoresistant osteosarcoma cells. Cancer Res. 2019;79(12): 3088-3099. doi:10.1158/0008-5472.CAN-18-2693

25. Xia WK, Liu ZL, Shen D, Lin QF, Su J, Mao WD. Prognostic performance of pre-treatment NLR and PLR in patients suffering from osteosarcoma. World J Surg Oncol. 2016;14:127. doi:10.1186/ s12957-016-0889-2

26. Chen H, Liu J, Cheng Z, et al. Development and external validation of an MRI-based radiomics nomogram for pretreatment prediction for early relapse in osteosarcoma: a retrospective multicenter study. Eur J Radiol. 2020;129:109066. doi:10.1016/j.ejrad.2020.109066

27. Jettoo P, Tan G, Gerrand CH, Rankin KS. Role of routine blood tests for predicting clinical outcomes in osteosarcoma patients. J Orthop Surg. 2019;27(2):2309499019838293. doi:10.1177/2309499019838293
28. Keller S, Inai R, Sato S, et al. Thallium-201 uptake of giant cell tumor: one step toward the differential diagnosis to atypically presenting osteosarcoma. AJR Am J Roentgenol. 2017;208(1):171-179. doi:10.2214/AJR.16.16359

29. Kerr KF, Brown MD, Zhu K, Janes H. Assessing the clinical impact of risk prediction models with decision curves: guidance for correct interpretation and appropriate use. J Clin Oncol. 2016;34(21):25 34-2540. doi:10.1200/JCO.2015.65.5654

30. Vickers AJ, Elkin EB. Decision curve analysis: a novel method for evaluating prediction models. Med Decis Making. 2006;26(6): 565-574. doi:10.1177/0272989X06295361

31. Misaghi A, Goldin A, Awad M, Kulidjian AA. Osteosarcoma: a comprehensive review. SICOT J. 2018;4:12. doi:10.1051/sicotj/ 2017028

\section{Publish your work in this journal}

The International Journal of General Medicine is an international, peer-reviewed open-access journal that focuses on general and internal medicine, pathogenesis, epidemiology, diagnosis, monitoring and treatment protocols. The journal is characterized by the rapid reporting of reviews, original research and clinical studies across all disease areas. The manuscript management system is completely online and includes a very quick and fair peer-review system, which is all easy to use. Visit http://www.dovepress.com/ testimonials.php to read real quotes from published authors. 